\title{
EFFICIENT AND SAFE GLOBAL CONSTRAINTS FOR HANDLING NUMERICAL CONSTRAINT SYSTEMS*
}

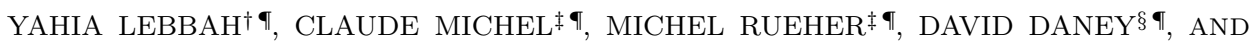 \\ JEAN-PIERRE MERLET $\S$
}

\begin{abstract}
Numerical constraint systems are often handled by branch and prune algorithms that combine splitting techniques, local consistencies, and interval methods. This paper first recalls the principles of Quad, a global constraint that works on a tight and safe linear relaxation of quadratic subsystems of constraints. Then, it introduces a generalization of Quad to polynomial constraint systems. It also introduces a method to get safe linear relaxations and shows how to compute safe bounds of the variables of the linear constraint system. Different linearization techniques are investigated to limit the number of generated constraints. QuadSolver, a new branch and prune algorithm that combines Quad, local consistencies, and interval methods, is introduced. QuadSolver has been evaluated on a variety of benchmarks from kinematics, mechanics, and robotics. On these benchmarks, it outperforms classical interval methods as well as constraint satisfaction problem solvers and it compares well with state-of-the-art optimization solvers.
\end{abstract}

Key words. systems of equations and inequalities, constraint programming, reformulation linearization technique, global constraint, interval arithmetic, safe rounding

AMS subject classifications. 65H10, 65G40,65H20, 93B18, 65G20

DOI. $10.1137 / \mathrm{S} 0036142903436174$

1. Introduction. Many applications in engineering sciences require finding all isolated solutions to systems of constraints over real numbers. These systems may be nonpolynomial and are difficult to solve: the inherent computational complexity is NP-hard and numerical issues are critical in practice (e.g., it is far from being obvious to guarantee correctness and completeness as well as to ensure termination). These systems, called numerical CSP (constraint satisfaction problem) in the rest of this paper, have been approached in the past by different interesting methods: ${ }^{1}$ interval methods [35, 24, 38, 20, 40], continuation methods [37, 2, 62], and constraint satisfaction methods $[30,6,11,61]$. Of particular interest is the mathematical and programming simplicity of the latter approach: the general framework is a branch and prune algorithm that requires only specifying the constraints and the initial range of the variables.

The purpose of this paper is to introduce and study a new branch and bound algorithm called QuadSolver. The essential feature of this algorithm is a global constraint - called Quad - that works on a tight and safe linear relaxation of the polynomial relations of the constraint systems. More precisely, QuadSolver is a branch

${ }^{*}$ Received by the editors October 15, 2003; accepted for publication (in revised form) June 3, 2004; published electronically February 25, 2005.

http://www.siam.org/journals/sinum/42-5/43617.html

†Département Informatique, Faculté des Sciences, Université d'Oran Es-Senia, BP 1524, ElM’Naouar Oran, Algeria (Yahia.Lebbah@sophia.inria.fr).

${ }^{\ddagger}$ Université de Nice-Sophia Antipolis, I3S-CNRS, 930 route des Colles, BP 145, 06903 Sophia Antipolis Cedex, France (Claude.Michel@sophia.inria.fr, rueher@essi.fr).

§INRIA, 2004 route des Lucioles, BP 93, 06902 Sophia Antipolis Cedex, France (David.Daney@ sophia.inria.fr, Jean-Pierre.Merlet@sophia.inria.fr).

๑ COPRIN Project INRIA-I3S-CNRS, 2004 route des Luciales, BP 93, Sophia Antipolis Cedex 06903, France.

${ }^{1}$ Alternative methods have been proposed for solving nonlinear systems. For instance, algebraic constraints can be handled with symbolic methods [13] (e.g., Groebner basis, resultant). However, these methods can neither handle nonpolynomial systems nor deal with inequalities. 
and prune algorithm that combines Quad, local consistencies, and interval methods. That is to say, QuadSolver is an attempt to merge the best interval and constraint programming techniques. QuadSolver has been evaluated on a variety of benchmarks from kinematics, mechanics, and robotics. On these benchmarks, it outperforms classical interval methods as well as CSP solvers and it compares well with state-of-the-art optimization solvers.

The Quad-filtering algorithm [27] has first been defined for quadratic constraints. The relaxation of quadratic terms is adapted from a classical linearization method, the reformulation-linearization technique (RLT) $[54,53]$. The simplex algorithm is used to narrow the domain of each variable with respect to the subset of the linear set of constraints generated by the relaxation process. The coefficients of these linear constraints are updated with the new values of the bounds of the domains and the process is restarted until no more significant reduction can be done. We have demonstrated [27] that the Quad algorithm yields a more effective pruning of the domains than local consistency filtering algorithms (e.g., 2b-consistency [30] or box-consistency [6]). Indeed, the drawback of classical local consistencies comes from the fact that the constraints are handled independently and in a blind way. ${ }^{2}$ That is to say, classical local consistencies do not exploit the semantic of quadratic terms; in other words, these approaches do not take advantage of the very specific semantic of quadratic constraints to reduce the domains of the variables. Conversely, linear programming techniques $[1,54,3]$ do capture most of the semantics of quadratic terms, e.g., convex and concave envelopes of these particular terms. ${ }^{3}$

The extension of Quad for handling any polynomial constraint system requires replacing nonquadratic terms by new variables and adding the corresponding identities to the initial constraint system. However, a complete quadrification [58] would generate a huge number of linear constraints. Thus, we introduce here an heuristic based on a good tradeoff between a tight approximation of the nonlinear terms and the size of the generated constraint system. This heuristic works well on classical benchmarks (see section 8).

A safe rounding process is a key issue for the Quad framework. Let us recall that the simplex algorithm is used to narrow the domain of each variable with respect to the subset of the linear set of constraints generated by the relaxation process. The point is that most implementations of the simplex algorithm are unsafe. Moreover, the coefficients of the generated linear constraints are computed with floating point numbers. So, two problems may occur in the Quad-filtering process.

1. The whole linearization may become incorrect due to rounding errors when computing the coefficients of the generated linear constraints.

2. Some solutions may be lost when computing the bounds of the domains of the variables with the simplex algorithm.

We propose in this paper a safe procedure for computing the coefficients of the generated linear constraints. Neumaier and Shcherbina [42] have addressed the second

\footnotetext{
${ }^{2} 3 \mathrm{~b}-$ consistency and kb-consistency are partial consistencies that can achieve a better pruning since they are "less local" [11]. However, they require numerous splitting steps to find the solutions of a system of quadratic constraints; so, they may become rather slow.

${ }^{3}$ Sherali and Tuncbilek [55] have also proposed four different filtering techniques for solving quadratic problems. Roughly speaking, the first filtering strategy performs a feasibility check on inequality constraints to discard subintervals of the domains of the variables. This strategy is very close to box-consistency filtering (see [60]). The three other techniques are based on specific properties of optimization problems with a quadratic objective function: the eigenstructure of the quadratic objective function, fathoming node, and Lagrangian dual problem. Thus, these techniques can be considered as local consistencies for optimization problems (see also [59] and Neumaier's survey [41]).
} 
problem. ${ }^{4}$ They have proposed a simple and cheap procedure to get a rigorous upper bound of the objective function. The incorporation of these procedures in the Quadfiltering process allows us to call the simplex algorithm without worrying about safety. So, with these two procedures, linear programming techniques can be used to tackle continuous CSPs without losing any solution.

The rest of this paper is organized as follows. Section 2 gives an overview of the approach whereas section 3 contains the notation. Sections 4 and 5 recall the basics of interval programming and constraint programming. Section 6 details the principle of the Quad algorithm, the linearization process, and the extension to polynomial constraints. Section 7 introduces the rounding process we propose to ensure the safe relaxations. Section 8 describes the experimental results and discusses related work. Concluding remarks are given in section 9 .

2. Overview of the approach. As mentioned, QuadSolver is a branch and prune algorithm that combines Quad and a box-consistency.

Box-consistency is the most successful adaptation of arc-consistency [31] to constraints over the real numbers. The box-consistency implementation of VanHentenryck, McAllester, and Kapur [60] is computed on three-interval extensions of the initial constraints: the natural interval extension, the distributed interval extension, and the Taylor interval extension with a conditioning step. The leftmost and the rightmost zeros are computed using a variation of the univariate interval Newton method.

The QuadSolver we propose here combines Quad-filtering and box-consistency filtering to prune the domain of the variables of numerical constraint systems. Operationally, QuadSolver performs the following filtering processes:

1. box-consistency filtering,

2. Quad-filtering.

The box-consistency is first used to detect some inconsistencies before starting the Quad-filtering algorithm which is more costly. These two steps are wrapped into a classical fixed point algorithm which stops when the domains of the variables cannot be further reduced. ${ }^{5}$

To isolate the different solutions, Quad uses classical branching techniques.

Before going into the details, let us outline the advantages of our approach on a couple of small examples.

2.1. Quad-filtering. Consider the constraint system $\mathcal{C}=\{2 x y+y=1, x y=0.2\}$ which represents two intersecting curves (see Figure 2.1). Suppose that $\mathbf{x}=[-10,+10]$ and $\mathbf{y}=[-10,+10]$ are the domains of the variables $x$ and $y$. An interval $\mathbf{x}=[\underline{x}, \bar{x}]$ denotes the set of reals $\{r \mid \underline{x} \leq r \leq \bar{x}\}$.

The RLT (see section 6.2) yields the following constraint system:

$$
\left\{\begin{array}{l}
y+2 w=1, \quad w=0.2, \\
\underline{y} x+\underline{x} y-w \leq \underline{x} \underline{y}, \quad \bar{y} x+\underline{x} y-w \geq \underline{x} \bar{y}, \\
\underline{y} x+\bar{x} y-w \geq \bar{x}, \quad \bar{y} x+\bar{x} y-w \leq \bar{x} y, \\
x \geq \underline{x}, \quad x \leq \bar{x}, \quad y \geq \underline{y}, \quad y \leq \bar{y},
\end{array}\right.
$$

where $w$ is a new variable that stands for the product $x y$. Note that constraint system (a) implies that $w \in[\underline{x}, \bar{x}] *[\underline{y}, \bar{y}]$.

\footnotetext{
${ }^{4}$ They have also suggested a solution to the first problem though their solution is dedicated to mixed integer programming problems.

${ }^{5}$ In practice, the loop stops when the domain reduction is lower than a given $\epsilon$.
} 


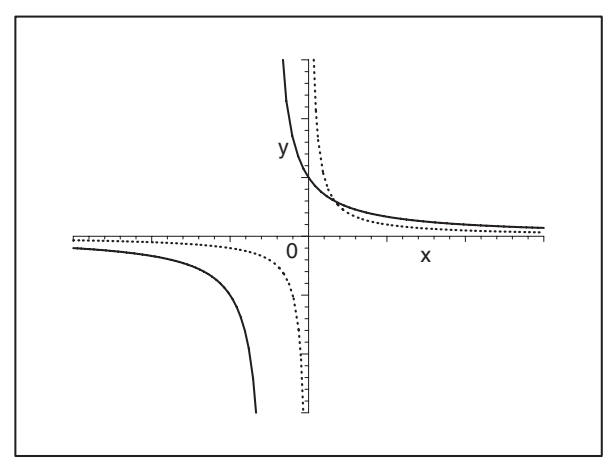

FIG. 2.1. Geometrical representation of $\{2 x y+y=1, x y=0.2\}$.

Substituting $\underline{x}, \underline{y}, \bar{x}$, and $\bar{y}$ by their values and minimizing (resp., maximizing) $x, y$, and $w$ with the simplex algorithm yield the following new bounds:

$$
\mathbf{x}=[-9.38,9.42], \quad \mathbf{y}=[0.6,0.6], \quad \mathbf{w}=[0.2,0.2] .
$$

By substituting the new bounds of $x, y$, and $w$ in the constraint system (a), we obtain a new linear constraint system. One more minimizing (resp., maximizing) step is required to obtain tight bounds of $\mathbf{x}$. Note that numerous splitting operations are required to find the unique solution of the problem with a 3b-consistency filtering algorithm. The proposed algorithm solves the problem by generating 6 linear constraints and with 8 calls to the simplex algorithm. It finds the same solution as a solver based on 3b-consistency but without splitting and in less time.

2.2. A safe rounding procedure. Consider the constraint system

$$
\mathcal{C}=\left\{\begin{array}{l}
w_{1}+w_{2}=1, \quad w_{1} x_{1}+w_{2} x_{2}=0 \\
w_{1} x_{1} x_{1}+w_{2} x_{2} x_{2}=1, \quad w_{1} x_{1} x_{1} x_{1}+w_{2} x_{2} x_{2} x_{2}=0
\end{array}\right.
$$

which represents a simple Gaussian quadrature formula to compute integrals [9]. Suppose that the domains of variables $x_{1}, x_{2}, w_{1}$, and $w_{2}$ are all equal to $[-1,+1]$. This system has two solutions:

- $x_{1}=-1, x_{2}=1, w_{1}=0.5, w_{2}=0.5$,

- $x_{1}=1, x_{2}=-1, w_{1}=0.5, w_{2}=0.5$.

A straightforward implementation of Quad would only find one unsafe solution with

$$
x_{2} \in[+0.9999 \ldots 944,+0.9999 \ldots 989] .
$$

Indeed, when we examine the Quad-filtering process, we can identify some linear programs where the simplex algorithm steps to the wrong side of the objective.

With the corrections we propose in section 7 , we obtain a tight approximation of the two correct solutions (with $x_{2} \in[-1.000000 \ldots,-0.999999 \ldots]$ and $x_{2} \in$ $[0.999999 \ldots, 1.000000 \ldots])$.

3. Notation and basic definitions. This paper focuses on CSPs where the domains are intervals and the constraints $C_{j}\left(x_{1}, \ldots, x_{n}\right)$ are $n$-ary relations over the reals. $\mathcal{C}$ stands for the set of constraints.

$\mathrm{x}$ or $D_{x}$ denotes the domain of variable $x$, that is to say, the set of allowed values for $x$. $\mathcal{D}$ stands for the set of domains of all the variables of the considered constraint 
system. $\mathbb{R}$ denotes the set of real numbers whereas $\mathbb{F}$ stands for the set of floating point numbers used in the implementation of nonlinear constraint solvers; if $a$ is a constant in $\mathbb{F}, a^{+}$(resp., $a^{-}$) corresponds to the smallest (resp., largest) number of $\mathbb{F}$ strictly greater (resp., lower) than $a$.

$\mathbf{x}=[\underline{x}, \bar{x}]$ is defined as the set of real numbers $x$ verifying $\underline{x} \leq x \leq \bar{x} . x, y$ denote real variables, $X, Y$ denote vectors whereas $\mathbf{X}, \mathbf{Y}$ denote interval vectors. The width $w(\mathbf{x})$ of an interval $\mathbf{x}$ is the quantity $\bar{x}-\underline{x}$ while the midpoint $m(\mathbf{x})$ of the interval $\mathrm{x}$ is $(\bar{x}+\underline{x}) / 2$. A point interval $\mathbf{x}$ is obtained if $\underline{x}=\bar{x}$. A box is a set of intervals: its width is defined as the largest width of its interval members, while its center is defined as the point whose coordinates is the midpoint of the ranges. $\mathbb{I}^{n}$ denotes the set of boxes and is ordered by set inclusion.

We use the RLT notation introduced in $[54,3]$ with slight modifications. More precisely, we will use the following notations: $[c]_{L}$ is the set of linear constraints generated by replacing the nonlinear terms by new variables in constraint $c$, and $[c]_{L I}$ denotes the set of equations that keep the link between the new variables and the nonlinear terms while $[c]_{R}$ contains linear inequalities that approximate the semantics of nonlinear terms of constraint $c$. These notations will be used indifferently whether $c$ is a constraint or $\mathcal{C}$ is a set of constraints.

Rounding is necessary to close the operations over $\mathbb{F}$ (see [18]). A rounding function maps the result of the evaluation of an expression to available floating-point numbers. Rounding $x$ towards $+\infty$ maps $x$ to the least floating point number $x_{f}$ such that $x \leq x_{f} . \nabla(x)$ (resp., $\triangle(x)$ ) denotes a rounding mode of $x$ towards $-\infty$ (resp., $+\infty$ ).

4. Interval programming. This section recalls the basic concepts of interval arithmetic that are required to understand the rest of the paper. Readers familiar with interval arithmetic may skip this section.

4.1. Interval arithmetic. Interval arithmetic has been introduced by Moore [35]. It is based on the representation of variables as intervals.

Let $f$ be a real-valued function of $n$ unknowns $X=\left(x_{1}, \ldots, x_{n}\right)$. An interval evaluation of $f$ for given ranges $\mathbf{X}=\left(\mathbf{x}_{1}, \ldots, \mathbf{x}_{n}\right)$ for the unknowns is an interval $\mathbf{y}$ such that

$$
\underline{y} \leq f(\mathbf{X}) \leq \bar{y} \text { for all } X=\left(x_{1}, \ldots, x_{n}\right) \in \mathbf{X}=\left(\mathbf{x}_{1}, \ldots, \mathbf{x}_{n}\right) .
$$

In other words, $\underline{y}$ and $\bar{y}$ are lower and upper bounds for the values of $f$ when the values of the unknowns are restricted to the box $\mathbf{X}$.

There are numerous ways to calculate an interval evaluation of a function [20,46]. The simplest is the natural evaluation in which all the mathematical operators in $f$ are substituted by their interval equivalents. Interval equivalents exist for all classical mathematical operators. Hence interval arithmetic allows us to calculate an interval evaluation for all nonlinear expressions, whether algebraic or not. For example, if $f(x)=x+\sin (x)$, then the interval evaluation of $f$ for $x \in[1.1,2]$ can be calculated as follows:

$$
f([1.1,2])=[1.1,2]+\sin ([1.1,2])=[1.1,2]+[0.8912,1]=[1.9912,3] .
$$

Interval arithmetic can be implemented with directed rounding to take into account round-off errors. There are numerous interval arithmetic packages implementing this property: one of the most famous library is BIAS/Profil,${ }^{6}$ but a promising new

\footnotetext{
${ }^{6}$ http://www.ti3.tu-harburg.de/Software/PROFILEnglisch.html.
} 
package — based on the multiprecision software $\mathrm{MPFR}^{7}$ — is MPFI [47].

The main limitation of interval arithmetic is the overestimation of interval functions. This is due to two well-known problems:

- the so-called wrapping effect [35, 39], which overestimates by a unique vector the image of an interval vector (which is in general not a vector). That is to say, $\{f(X) \mid X \in \mathbf{X}\}$ is contained in $f(\mathbf{X})$ but is usually not equal to $f(\mathbf{X})$;

- the so-called dependency problem [20], which is due to the independence of the different occurrences of some variables during the interval evaluation of an expression. In other words, during the interval evaluation process there is no correlation between the different occurrences of a same variable in an equation. For instance, consider $\mathbf{x}=[0,10] . \mathbf{x}-\mathbf{x}=[\underline{x}-\bar{x}, \bar{x}-\underline{x}]=[-10,10]$ instead of $[0,0]$ as one could expect.

In general, it is not possible to compute the exact enclosure of the range for an arbitrary function over the real numbers [25]. Thus, Moore introduced the concept of interval extension: the interval extension of a function is an interval function that computes outer approximations on the range of the function over a domain [20,36]. Two main extensions have been introduced: the natural extension and the Taylor extension $[46,20,38] .{ }^{8}$ Due to the properties of interval arithmetic, the evaluation of a function may yield different results according to the literal form of the equations. Thus, many literal forms may be used as, for example, factorized form (Horner for polynomial system) or distributed form [60].

Nevertheless, in general, neither the natural form nor the Taylor expansion allows us to compute the exact range of a function $f$. For instance, considering $f(x)=$ $1-x+x^{2}$ and $\mathbf{x}=[0,2]$, we have

$$
\begin{aligned}
f_{\text {tay }}([0,2]) & =f(x)+(2 \mathbf{x}-1)(\mathbf{x}-x)=f(1)+(2[0,2]-1)([0,2]-1)=[-2,4], \\
f([0,2]) & =1-\mathbf{x}+\mathbf{x}^{2}=1-[0,2]+[0,2]^{2}=[-1,5] \\
f_{\text {factor }}([0,2]) & =1+\mathbf{x}(\mathbf{x}-1)=1+[0,2]([0,2]-1)=[-1,3]
\end{aligned}
$$

whereas the range of $f$ over $X=[0,2]$ is $[3 / 4,3]$. In this case, this result could directly be obtained by a second form of factorization: $f_{\text {factor }_{2}}([0,2])=(\mathbf{x}-1 / 2)^{2}+3 / 4=$ $([0,2]-1 / 2)^{2}+3 / 4=[3 / 4,3]$.

4.2. Interval analysis methods. This section provides a short introduction to interval analysis methods (see [35, 20, 38, 40] for a more detailed introduction). We limit this overview to interval Newton-like methods for solving a multivariate system of nonlinear equations. Their use is complementary to methods provided by the constraint programming community.

The aim is to determine the zeros of a system of $n$ equations $f_{i}\left(x_{1}, \ldots, x_{n}\right)$ in $n$ unknowns $x_{i}$ inside the interval vector $\mathbf{X}=\left(\mathbf{x}_{1}, \ldots, \mathbf{x}_{n}\right)$ with $x_{i} \in \mathbf{x}_{i}$ for $i=1, \ldots, n$.

First, consider solving an interval linear system of equations defined as follows:

$$
A X=b, \quad A \in \mathbf{A}, b \in \mathbf{b},
$$

where $\mathbf{A}$ is an interval matrix and $\mathbf{b}$ is an interval vector. Solving this linear interval system requires us to determine an interval vector $\mathbf{X}$ containing all solutions of all scalar linear systems noted $A X=b$ such that $A \in \mathbf{A}$ and $b \in \mathbf{b}$. Finding the exact value of $\mathbf{X}$ is a difficult problem, but three basic interval methods exist: Gaussian

\footnotetext{
${ }^{7}$ http://www.mpfr.org.

${ }^{8} f_{\text {tay }}(\mathbf{X})=f(X)+\mathbf{A}(\mathbf{X}-X)$, where $\mathbf{A}$ is the Jacobian or the interval slope matrix.
} 
elimination, Gauss-Seidel iterative method, or Krawczyk method (see [24, 38, 20, 40]). They may provide an overestimated interval vector $\mathbf{X}_{1}$ including $\mathbf{X}$. However, in general the computed intervals are too wide and a preconditioning is required, that is to say, a multiplication of both sides of (4.3) by the inverse of a midpoint of $\mathbf{A}$. The matrix $m(\mathbf{A})^{-1} \mathbf{A}$ is then "closer" to the identity matrix and the width of $\mathbf{X}_{1}$ is smaller [20].

To solve nonlinear systems, an interval Newton algorithm is often used-see [20] or [38]. The basic idea is to solve iteratively a linear approximation of the nonlinear system obtained by a Taylor expansion. Many improvements [24, 19], based on variations of the resolution of the linear subsystem or the preconditioning, have been proposed. Note that many interesting properties are provided by Newton-like methods: existence and/or uniqueness of a root, convergence area/rate, ....

5. Constraint programming. This section recalls the basics of constraint programming techniques which are required to understand the rest of this paper. A detailed discussion of these concepts and techniques can be found in [6, 26].

5.1. The general framework. The constraint programming framework is based on a branch and prune scheme which was inspired by the traditional branch and bound approach used in optimization problems. That is to say, it is best viewed as an iteration of two steps [60]:

1. pruning the search space;

2. making a choice to generate two (or more) subproblems.

The pruning step ensures that some local consistency holds. In other words, the pruning step reduces an interval when it can prove that the upper bound or the lower bound does not satisfy some constraint. Informally speaking, a constraint system $\mathcal{C}$ satisfies a partial consistency property if a relaxation of $\mathcal{C}$ is consistent. For instance consider $\mathbf{x}=[\underline{x}, \bar{x}]$ and $c\left(x, x_{1}, \ldots, x_{n}\right) \in \mathcal{C}$. Whenever $c\left(x, x_{1}, \ldots, x_{n}\right)$ does not hold for any values $a \in \mathbf{x}=\left[\underline{x}, x^{\prime}\right]$, then $\mathbf{x}$ may be shrunk to $\mathbf{x}=\left[x^{\prime}, \bar{x}\right]$. Local consistencies are detailed in the next subsection. Roughly speaking, they are relaxations of arcconsistency, a notion that is well known in artificial intelligence [31, 34].

The branching step usually splits the interval associated to some variable in two intervals with the same width. However, the splitting process may generate more than two subproblems and one may split an interval at a point different from its midpoint. The choice of the variable to split is a critical issue in difficult problems. Sophisticated splitting strategies have been developed for finite domains but few results [23] are available for continuous domains.

5.2. Local consistencies $[\mathbf{1 1}, \mathbf{2 6}]$. Local consistencies are conditions that filtering algorithms must satisfy. A filtering algorithm can be seen as a fixed point algorithm defined by the sequence $\left\{\mathcal{D}_{k}\right\}$ of domains generated by the iterative application of an operator $O p: \mathbb{R}^{n} \longrightarrow \mathbb{I}^{n}$ (see Figure 5.1).

$$
\mathcal{D}_{k}= \begin{cases}\mathcal{D} & \text { if } k=0 \\ O p\left(\mathcal{D}_{k-1}\right) & \text { if } k>0\end{cases}
$$

FIG. 5.1. Filtering algorithms as fixed point algorithms.

The operator $O p$ of a filtering algorithm generally satisfies the following three properties:

- $O p(\mathcal{D}) \subseteq \mathcal{D}$ (contractance);

- $O p$ is conservative; that is, it cannot remove any solution; 
- $\mathcal{D}^{\prime} \subseteq \mathcal{D} \Rightarrow O p\left(\mathcal{D}^{\prime}\right) \subseteq O p(\mathcal{D})$ (monotonicity).

Under those conditions, the limit of the sequence $\left\{\mathcal{D}_{k}\right\}$, which corresponds to the greatest fixed point of the operator $O p$, exists and is called a closure. A fixed point for $O p$ may be characterized by an $l c$-consistency property, called a local consistency. The algorithm achieving filtering by $l c$-consistency is denoted $l c$-filtering. A CSP is said to be $l c$-satisfiable if $l c$-filtering of this CSP does not produce an empty domain.

Consistencies used in numerical CSP solvers can be categorized in two main classes: arc-consistency-like consistencies and strong consistencies. Strong consistencies will not be discussed in this paper (see $[30,26]$ for a detailed introduction).

Most of the numerical CSP systems (for example, BNR-prolog [43], Interlog [8], CLP(BNR) [7], PrologIV [12], UniCalc [4], Ilog Solver [22], Numerica [61], and RealPaver [5]) compute an approximation of arc-consistency [31] which will be named ac-like-consistency in this paper. An ac-like-consistency states a local property on a constraint and on the bounds of the domains of its variables. Roughly speaking, a constraint $c_{j}$ is ac-like-consistent if for any variable $x_{i}$ in $\operatorname{var}\left(c_{j}\right)$, the bounds $\underline{x}_{i}$ and $\bar{x}_{i}$ have a support in the domains of all other variables of $c_{j}$.

The most famous ac-like consistencies are 2b-consistency and box-consistency.

2b-consistency (also known as hull consistency) [10, 7, 28, 30] requires only to check the arc-consistency property for each bound of the intervals. The key point is that this relaxation is more easily verifiable than arc-consistency itself. Informally speaking, variable $x$ is $2 \mathrm{~b}$-consistent for constraint " $f\left(x, x_{1}, \ldots, x_{n}\right)=0$ " if the lower (resp., upper) bound of the domain of $x$ is the smallest (resp., largest) solution of $f\left(x, x_{1}, \ldots, x_{n}\right)$. The box-consistency $[6,21]$ is a coarser relaxation (i.e., it allows less stringent pruning) of arc-consistency than 2b-consistency. Variable $x$ is box-consistent for constraint " $f\left(x, x_{1}, \ldots, x_{n}\right)=0$ " if the bounds of the domain of $x$ correspond to the leftmost and rightmost zeros of the optimal interval extension of $f\left(x, x_{1}, \ldots, x_{n}\right)$. 2b-consistency algorithms actually achieve a weaker filtering (i.e., a filtering that yields bigger intervals) than box-consistency, more precisely when a variable occurs more than once in some constraint (see Proposition 6 in [11]). This is due to the fact that $2 \mathrm{~b}$-consistency algorithms require a decomposition of the constraints with multiple occurrences of the same variable.

2b-consistency [30] states a local property on the bounds of the domains of a variable at a single constraint level. A constraint $c$ is $2 \mathrm{~b}$-consistent if, for any variable $x$, there exist values in the domains of all other variables which satisfy $c$ when $x$ is fixed to $\underline{x}$ and $\bar{x}$.

The filtering by 2b-consistency of $P=(\mathcal{D}, \mathcal{C})$ is the CSP $P^{\prime}=\left(\mathcal{D}^{\prime}, \mathcal{C}\right)$ such that

- $P$ and $P^{\prime}$ have the same solutions;

- $P^{\prime}$ is 2 b-consistent;

- $\mathcal{D}^{\prime} \subseteq \mathcal{D}$ and the domains in $\mathcal{D}^{\prime}$ are the largest ones for which $P^{\prime}$ is $2 \mathrm{~b}-$ consistent.

Filtering by 2b-consistency of $P$ always exists and is unique [30], that is to say it is a closure.

The box-consistency $[6,21]$ is a coarser relaxation of arc-consistency than 2bconsistency. It mainly consists of replacing every existentially quantified variable but one with its interval in the definition of $2 \mathrm{~b}$-consistency. Thus, box-consistency generates a system of univariate interval functions which can be tackled by numerical methods such as interval Newton. In contrast to 2b-consistency, box-consistency does not require any constraint decomposition and thus does not amplify the locality problem. Moreover, box-consistency can tackle some dependency problems when each 
constraint of a CSP contains only one variable which has multiple occurrences. More formally we have the following definition.

Definition 5.1 (box-consistency). Let $(\mathcal{D}, \mathcal{C})$ be a CSP and $c \in \mathcal{C}$ a $k$-ary constraint over the variables $\left(x_{1}, \ldots, x_{k}\right)$. $c$ is box-consistent if, for all $x_{i}$, the following relations hold:

1. $c\left(\mathbf{x}_{1}, \ldots, \mathbf{x}_{i-1},\left[\underline{x}_{i}, \underline{x}_{i}^{+}\right), \mathbf{x}_{i+1}, \ldots, \mathbf{x}_{k}\right)$,

2. $c\left(\mathbf{x}_{1}, \ldots, \mathbf{x}_{i-1},\left(\bar{x}_{i}^{-}, \bar{x}_{i}\right], \mathbf{x}_{i+1}, \ldots, \mathbf{x}_{k}\right)$.

Closure by box-consistency of $P$ is defined similarly as closure by 2 b-consistency of $P$.

Benhamou et al. have introduced HC4 [5], an ac-like-consistency that merges $2 \mathrm{~b}$-consistency and box-consistency and which optimizes the computation process.

6. Quad basics and extensions. This section first introduces Quad, a global constraint that works on a tight and safe linear relaxation of quadratic subsystems of constraints. Then, it generalizes Quad to the polynomial part of numerical constraint systems. Different linearization techniques are investigated to limit the number of generated constraints.

6.1. The Quad algorithm. The Quad-filtering algorithm (see Algorithm 1) consists of three main steps: reformulation, linearization, and pruning.

The reformulation step generates $[\mathcal{C}]_{R}$, the set of implied linear constraints. More precisely, $[\mathcal{C}]_{R}$ contains linear inequalities that approximate the semantics of nonlinear terms of $\mathcal{C}$.

The linearization process first decomposes each nonlinear term in sums and products of univariate terms; then it replaces nonlinear terms with their associated new variables. For example, considering constraint $c: x_{2} x_{3} x_{4}^{2}\left(x_{6}+x_{7}\right)+\sin \left(x_{1}\right)\left(x_{2} x_{6}-\right.$ $\left.x_{3}\right)=0$, a simple linearization transformation may yield the following sets:

- $[c]_{L}=\left\{y_{1}+y_{3}=0, \quad y_{2}=x_{6}+x_{7}, y_{4}=y_{5}-x_{3}\right\}$,

- $[c]_{L I}=\left\{y_{1}=x_{2} x_{3} x_{4}^{2} y_{2}, y_{3}=\sin \left(x_{1}\right) y_{4}, y_{5}=x_{2} x_{6}\right\}$.

$[c]_{L}$ is the set of linear constraints generated by replacing the nonlinear terms by new variables and $[c]_{L I}$ denotes the set of equations that keep the link between the new variables and the nonlinear terms. Note that the nonlinear terms which are not directly handled by the Quad are taken into account by the box-filtering process.

Finally, the linearization step computes the set of final linear inequalities and equations $L R=[\mathcal{C}]_{L} \cup[\mathcal{C}]_{R}$, the linear relaxation of the original constraints $\mathcal{C}$.

The pruning step is just a fixed point algorithm that calls iteratively a linear programming solver to reduce the upper and lower bounds of every original variable. The algorithm converges and terminates if $\epsilon$ is greater than zero.

Now we are in the position to introduce the reformulation of nonlinear terms. Section 6.2 first introduces the handling of quadratic constraints while section 6.3 extends the previous results to polynomial constraints.

6.2. Handling quadratic constraints. Quadratic constraints are approximated by linear constraints in the following way. Quad creates a new variable for each quadratic term: $y$ for $x^{2}$ and $y_{i, j}$ for $x_{i} x_{j}$. The produced system is denoted as

$$
\left[\sum_{(i, j) \in M} a_{k, i, j} x_{i} x_{j}+\sum_{i \in N} b_{k, i} x_{i}^{2}+\sum_{i \in N} d_{k, i} x_{i}=b_{k}\right]_{L} .
$$




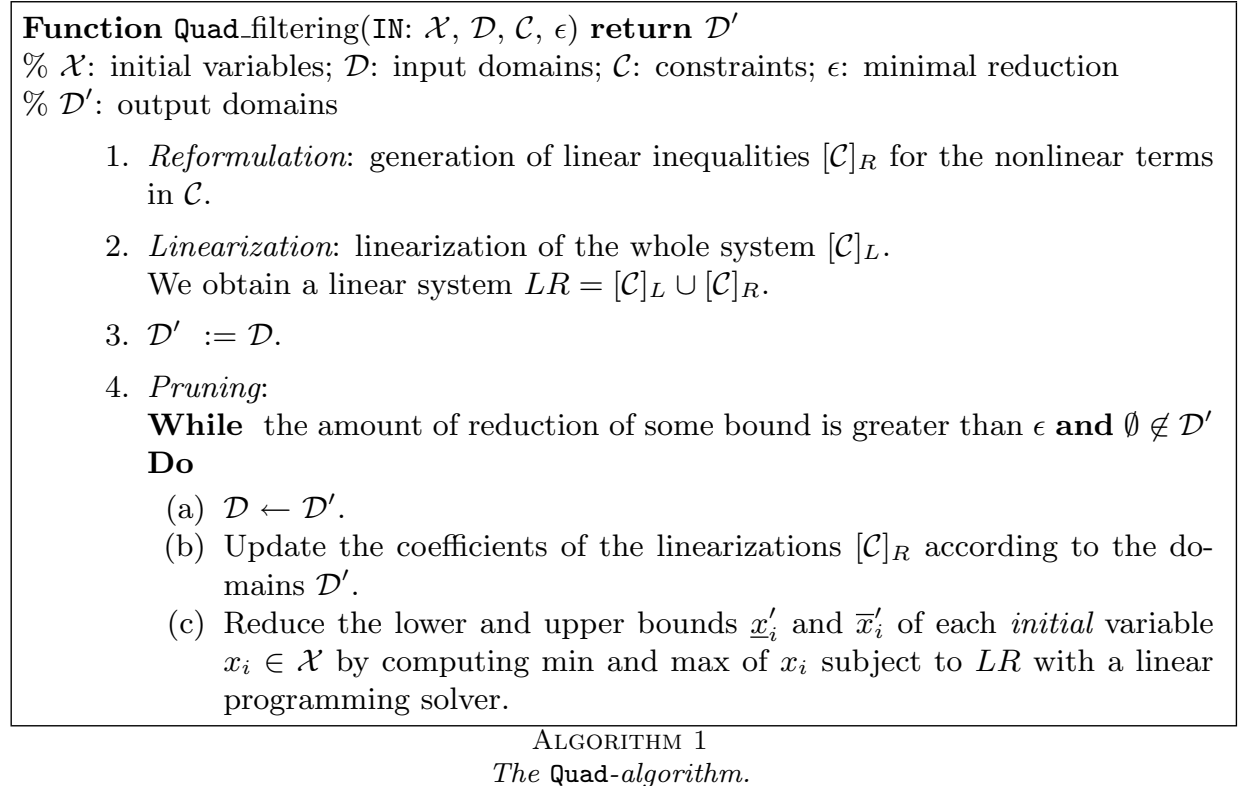

A tight linear (convex) relaxation, or outer-approximation to the convex and concave envelope of the quadratic terms over the constrained region, is built by generating new linear inequalities.

Quad uses two tight linear relaxation classes that preserve equations $y=x^{2}$ and $y_{i, j}=x_{i} x_{j}$ and that provide a better approximation than interval arithmetic [27].

6.2.1. Linearization of $\boldsymbol{x}^{2}$. The term $x^{2}$ with $\underline{x} \leq x \leq \bar{x}$ is approximated by the following relations:

$$
\left[x^{2}\right]_{R}=\left\{\begin{aligned}
L 1(\alpha) & \equiv\left[(x-\alpha)^{2} \geq 0\right]_{L}, \quad \text { where } \alpha \in[\underline{x}, \bar{x}], \\
L 2 & \equiv[(\underline{x}+\bar{x}) x-y-\underline{x} \bar{x} \geq 0]_{L} .
\end{aligned}\right.
$$

Note that $\left[\left(x-\alpha_{i}\right)^{2}=0\right]_{L}$ generates the tangent line to the curve $y=x^{2}$ at the point $x=\alpha_{i}$. Actually, Quad computes only $L 1(\bar{x})$ and $L 1(\underline{x})$. Consider for instance the quadratic term $x^{2}$ with $x \in[-4,5]$. Figure 6.1 displays the initial curve (i.e., $\left.D_{1}\right)$ and the lines corresponding to the equations generated by the relaxations: $D_{2}$ for $L 1(-4) \equiv y+8 x+16 \geq 0, D_{3}$ for $L 1(5) \equiv y-10 x+25 \geq 0$, and $D_{4}$ for $L 2 \equiv-y+x+20 \geq 0$.

We may note that $L 1(\bar{x})$ and $L 1(\underline{x})$ are underestimations of $x^{2}$ whereas $L 2$ is an overestimation. $L 2$ is also the concave envelope, which means that it is the optimal concave overestimation.

6.2.2. Bilinear terms. In the case of bilinear terms $x y$, McCormick [32] proposed the following relaxations of $x y$ over the box $[\underline{x}, \bar{x}] \times[\underline{y}, \bar{y}]$, stated in the equivalent RLT form [54]:

$$
[x y]_{R}=\left\{\begin{array}{l}
B I L 1 \equiv[(x-x)(y-y) \geq 0]_{L} \\
B I L 2 \equiv[(x-\underline{x})(\bar{y}-\bar{y}) \geq 0]_{L} \\
B I L 3 \equiv[(\bar{x}-x)(y-y) \geq 0]_{L} \\
B I L 4 \equiv[(\bar{x}-x)(\bar{y}-\bar{y}) \geq 0]_{L}
\end{array}\right.
$$




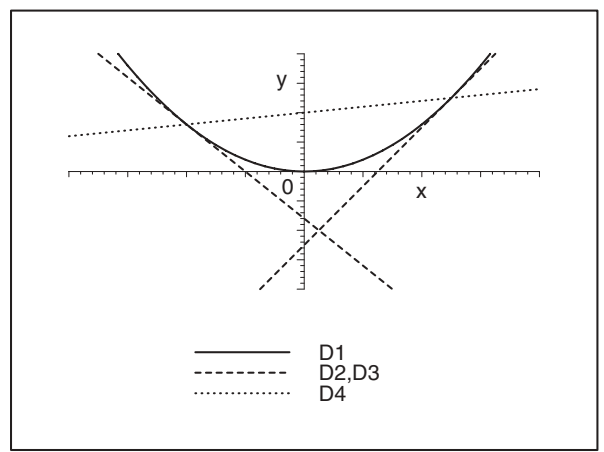

FIG. 6.1. Approximation of $x^{2}$.

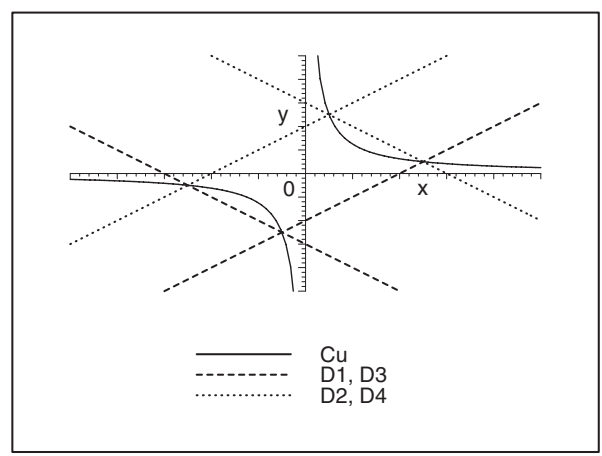

FIG. 6.2. Illustration of $x y$ relaxations.

$B I L 1$ and $B I L 3$ define a convex envelope of $x y$ whereas BIL2 and BIL4 define a concave envelope of $x y$ over the box $[\underline{x}, \bar{x}] \times[\underline{y}, \bar{y}]$. Al-Khayyal and Falk [1] showed that these relaxations are the optimal convex/concave outer-estimations of $x y$.

Consider for instance the quadratic term $x y$ with $x \in[-5,5]$ and $y \in[-5,5]$. The work done by the linear relaxations of the three-dimensional curve $z=x y$ is well illustrated in two dimensions by fixing $z$. Figure 6.2 displays the two-dimensional shape, for the level $z=5$, of the initial curve (i.e., $\mathrm{Cu}$ ) and the lines corresponding to the equations generated by the relaxations (where $z=5$ ): $D_{1}$ for $B I L 1 \equiv z+5 x+5 y+$ $25 \geq 0, D_{2}$ for $B I L 2 \equiv-z+5 x-5 y+25 \geq 0, D_{3}$ for $B I L 3 \equiv-z-5 x+5 y+25 \geq 0$, and $D_{4}$ for $B I L 4 \equiv z-5 x-5 y+25 \geq 0$.

6.3. Extension to polynomial constraints. In this section, we show how to extend the linearization process to polynomial constraints. We first discuss the quadrification process and compare it with RLT. Then, we present the linearizations of product and power terms.

6.3.1. Transformation of nonlinear constraints into quadratic constraints. In this section, we show how to transform a polynomial constraint system into an equivalent quadratic constraint system, a process called quadrification [58].

For example, considering the constraint $c: x_{2} x_{3} x_{4}^{2}+3 x_{6} x_{7}+\sin \left(x_{1}\right)=0$, the proposed transformation yields

$$
\left\{y_{1} y_{2}+3 y_{2}+s_{1}=0, \quad y_{1}=x_{2} x_{3}, \quad y_{2}=x_{4} x_{4}, \quad y_{3}=x_{6} x_{7}\right\}
$$

and the set $\left\{y_{1}=x_{2} x_{3}, \quad y_{2}=x_{4}^{2}, \quad y_{3}=x_{6} x_{7}, \quad s_{1}=\sin \left(x_{1}\right)\right\}$ of equations that keep the link between the new variables and the nonlinear terms that cannot be further quadrified. Such a transformation is one of the possible quadrifications. It is called a single quadrification.

We could generate all possible single quadrifications, or all quadrifying identities, and perform a so-called complete quadrification. For example, the complete quadrification of $E=\left\{x_{2} x_{3} x_{4}^{2}+3 x_{6} x_{7}+\sin \left(x_{1}\right)=0\right\}$ is

$$
\left\{\begin{array}{l}
y_{1}+3 y_{2}+s_{1}=0, \quad y_{2}=x_{6} x_{7}, \\
y_{1}=y_{3} y_{4}, \quad y_{3}=x_{2} x_{3}, \quad y_{4}=x_{4}^{2}, \\
y_{1}=y_{5} y_{6}, \quad y_{5}=x_{2} x_{4}, \quad y_{6}=x_{3} x_{4}, \\
y_{1}=x_{2} y_{7}, \quad y_{7}=x_{3} y_{4}, \quad y_{7}=x_{4} y_{6}, \\
y_{1}=x_{3} y_{8}, \quad y_{8}=x_{2} y_{4}, \quad y_{8}=x_{4} y_{5}, \quad \\
y_{1}=x_{4} y_{9}, \quad y_{9}=x_{2} y_{6}, \quad y_{9}=x_{3} y_{5}, \quad y_{9}=x_{4} y_{3},
\end{array}\right.
$$


where $s_{1}=\sin \left(x_{1}\right)$.

A quadrification for polynomial problems was introduced by Shor [58]. Sherali and Tuncbilek [57] have proposed a direct reformulation/linearization (RLT) of the whole polynomial constraints without quadrifying the constraints. They did prove the dominance of their direct reformulation/linearization technique over Shor's quadrification [56].

A complete quadrification generates as many new variables as the direct RLT. Linearizations proposed in RLT are built on every nonordered combination of $\delta$ variables, where $\delta$ is the highest polynomial degree of the constraint system.

The complete quadrification generates linearizations on every couple of nonordered combined variables $\left[v_{i}, v_{j}\right]$ where $v_{i}$ (resp., $v_{j}$ ) is the variable that has been introduced for linearizing the nonordered combination of variables.

Complete quadrification and direct RLT yield a tighter linearization than the single quadrification but the number of generated linearizations grows in an exponential way for nontrivial polynomial constraint systems. More precisely, the number of linearizations depends directly on the number of generated new variables.

To sum up, the linearization of polynomial systems offers two main possibilities: the transformation of the initial problem into an equivalent quadratic constraint system through a process called quadrification, or the direct linearization of polynomial terms by means of RLT. Theoretical considerations, as well as experimentations, have been conducted to exclude as practical a complete quadrification which produces a huge amount of linear inequalities for nontrivial polynomial systems. The next two subsections present our choices for the linearization of product and power terms.

6.3.2. Product terms. For the product term

$$
x_{1} x_{2} \ldots x_{n}
$$

we use a two-step procedure: quadrification and bilinear relaxations.

Since many single quadrifications exist, an essential point is the choice of a good heuristic that captures most of the semantics of the polynomial constraints. We use a "middle" heuristic to obtain balanced degrees on the generated terms. For instance, considering $T \equiv x_{1} x_{2} \ldots x_{n}$, a monomial of degree $n$, the middle heuristic will identify two monomials $T_{1}$ and $T_{2}$ of highest degree such that $T=T_{1} T_{2}$. It follows that $T_{1}=x_{1} x_{2} \ldots x_{n \div 2}$ and $T_{1}=x_{n \div 2+1} \ldots x_{n}$.

The quadrification is performed by recursively decomposing each product $x_{i} \ldots x_{j}$ into two products $x_{i} \ldots x_{d}$ and $x_{d+1} \ldots x_{j}$. Of course, there are many ways to choose the position of $d$. Ryoo and Sahinidis [49] and Sahinidis and Twarmalani [51] use what they call rAI, "recursive interval arithmetic," which is a recursive quadrification where $d=j-1$. We use the middle heuristic Qmid, where $d=(i+j) / 2$, to obtain balanced degrees on the generated terms. Let us denote by $[E]_{R I}$ the set of equations that transforms a product terms into a set of quadratic identities.

The second step consists of a bilinear relaxation $\left[[\mathcal{C}]_{R I}\right]_{R}$ of all the quadratic identities in $[\mathcal{C}]_{R I}$ with the bilinear relaxations introduced in section 6.2.2.

Sherali and Tuncbilek [57] have proposed a promising direct reformulation/ linearization technique (RLT) of the whole polynomial constraints without quadrifying the constraints. Applying RLT on the product term $x_{1} x_{2} \ldots x_{n}$ generates the 
following $n$-ary inequalities: ${ }^{9}$

$$
\prod_{i \in J_{1}}\left(x_{i}-\underline{x}_{i}\right) \prod_{i \in J_{2}}\left(\bar{x}_{i}-x_{i}\right) \geq 0 \text { for all } J_{1}, J_{2} \subseteq\{1, \ldots, n\}:\left|J_{1} \cup J_{2}\right|=n,
$$

where $\{1, \ldots, n\}$ is to be understood as a multiset and where $J_{1}$ and $J_{2}$ are multisets.

We now introduce Proposition 6.1, which states the number of new variables and relaxations, respectively, generated by the quadrification and RLT process on the product term (6.3).

Proposition 6.1. Let $T \equiv x_{1} x_{2} \ldots x_{n}$ be some product of degree $n \geq 1$ with $n$ distinct variables. The RLT of $T$ will generate up to $\left(2^{n}-n-1\right)$ new variables and $2^{n}$ inequalities whereas the quadrification of $T$ will generate only $(n-1)$ new variables and $4(n-1)$ inequalities.

Proof. The number of terms of length $i$ is clearly the number of combinations of $i$ elements within $n$ elements, that is to say $\mathrm{C}_{n}^{i}$. In the RLT relaxations (6.4), we generate new variables for all these combinations. Thus, the number of variables is bounded by $\sum_{i=2, \ldots, n} \mathrm{C}_{n}^{i}=\sum_{i=0, \ldots, n} \mathrm{C}_{n}^{i}-n-1$, that is to say $2^{n}-n-1$ since $\sum_{i=0, \ldots, n} \mathrm{C}_{n}^{i}=2^{n}$. In (6.4), for each variable we consider alternatively the lower bound and the upper bound: thus there are $2^{n}$ new inequalities.

For the quadrification process, the proof can be done by induction. For $n=1$, the formula is true. Now suppose that for length $i$ (with $1 \leq i<n),(i-1)$ new variables are generated. For $i=n$, we can split the term at the position $d$ with $1 \leq d<n$. It results from the induction hypothesis that we have $d-1$ new variables for the first part, and $n-d-1$ new variables for the second part, plus one more new variable for the whole term. So, $n-1$ new variables are generated. Bilinear terms require four relaxations, thus we get $4(n-1)$ new inequalities.

Proposition 6.2 states that quadrification with bilinear relaxations provides convex and concave envelopes with any $d$. This property results from the proof given in [49] for the rAI heuristic.

Proposition 6.2. $\left[\left[x_{1} x_{2} \ldots x_{n}\right]_{R I}\right]_{R}$ provides convex and concave envelopes of the product term $x_{1} x_{2} \cdots x_{n}$.

Generalization for sums of products, the so-called multilinear terms

$$
\sum_{i=1, \ldots, t} a_{i} \prod_{j \in J_{i}} x_{j}
$$

have been studied recently $[14,52,48,49]$. It is well known that finding the convex or concave envelope of a multilinear term is an NP-hard problem [14]. The most common method of linear relaxation of multilinear terms is based on the simple product term. However, it is also well known that this approach leads to a poor approximation of the linear bounding of the multilinear terms. Sherali [52] has introduced formulae for computing convex envelopes of the multilinear terms. It is based on an enumeration of vertices of a pre-specified polyhedra which is of exponential nature. Rikun [48] has given necessary and sufficient conditions for the polyhedrality of convex envelopes. He has also provided formulae of some faces of the convex envelope of a multilinear function. To summarize, it is difficult to characterize convex and concave envelopes for general multilinear terms. Conversely, the approximation of "product of variables" is an effective approach; moreover, it is easy to implement $[51,50]$.

\footnotetext{
${ }^{9}$ Linearizations proposed in RLT on the whole polynomial problem are built on every nonordered combination of $\delta$ variables, where $\delta$ is the highest polynomial degree of the constraint system.
} 
6.3.3. Power terms. A power term of the form $x^{n}$ can be approximated by $n+1$ inequalities with a procedure proposed by Sherali and Tuncbilek [57], called "bound-factor product RLT constraints." It is defined by the following formula:

$$
\left[x^{n}\right]_{R}=\left\{\left[(x-\underline{x})^{i}(\bar{x}-x)^{n-i} \geq 0\right]_{L}, i=0, \ldots, n\right\} .
$$

The essential observation is that this relaxation generates tight relations between variables on their upper and lower bounds. More precisely, suppose that some original variable takes a value equal to either of its bounds. Then all the corresponding new RLT linearization variables that involve this original variable take relative values that conform with actually fixing this original variable at its particular bound in the nonlinear expressions represented by these new RLT variables [57].

Note that relaxations (6.5) of the power term $x^{n}$ are expressed with $x^{i}$ for all $i \leq n$, and thus provide a fruitful relationship on problems containing many power terms involving some variable.

The univariate term $x^{n}$ is convex when $n$ is even, or when $n$ is odd and the value of $x$ is negative; it is concave when $n$ is odd and the value of $x$ is positive. Sahinidis and Twarmalani [50] have introduced the convex and concave envelopes when $n$ is odd by taking the point where the power term $x^{n}$ and its underestimator have the same slope. These convex/concave relaxations on $x^{n}$ are expressed with only $\left[x^{n}\right]_{L}$ and $x$. In other words, they do not generate any relations with $x^{i}$ for $1<i<n$.

That is why we suggest implementing the approximations defined by formulae (6.5). Note that for the case $n=2,(6.5)$ provides the concave envelope.

7. A safe rounding procedure for the Quad-algorithm. This section details the rounding procedure we propose to ensure the completeness of the Quad algorithm [33]. First, we show how to compute safe coefficients for the generated linear constraints. In the second subsection we explain how a recent result from Neumaier and Shcherbina [42] allows us to use the simplex algorithm in a safe way.

\subsection{Computing safe coefficients.}

(a) Approximation of $L 1$. The linear constraint $L 1(y, \alpha) \equiv y-2 \alpha x+\alpha^{2} \geq 0$ approximates a term $x^{2}$ with $\alpha \in[\underline{x}, \bar{x}] . L 1(y, \alpha)$ corresponds to the tangent lines to the curve $y=x^{2}$ at the point $\left(\alpha, \alpha^{2}\right)$.

Thus, the computation over the floats of the coefficients of $L 1(y, \alpha)$ may change the slope of the tangent line as well as the intersection points with the curve $y=x^{2}$. Consider the case where $\alpha$ is negative: the solutions are above the tangent line; thus we have to decrease the slope to be sure to keep all of the solutions. It follows that we have to use a rounding mode towards $+\infty$. Likewise, when $\alpha$ is positive, we have to set the rounding mode towards $-\infty$. More formally, we have

$$
L 1_{\mathbb{F}}(y, \alpha) \equiv \begin{cases}y-\nabla(2 \alpha) x+\triangle\left(\alpha^{2}\right) \geq 0 & \text { if } \alpha \geq 0 \\ y-\triangle(2 \alpha) x+\triangle\left(\alpha^{2}\right) \geq 0 & \text { if } \alpha<0\end{cases}
$$

where $\nabla(x)$ (resp., $\triangle(x)$ ) denotes a rounding mode of $x$ towards $-\infty$ (resp., $+\infty$ ).

(b) Approximation of $L 2$. The case of $L 2$ is a bit more tricky since the "rotation axis" of the line defined by $L 2$ is between the extremum values of $x^{2}(L 2(y)$ is an overestimation of $y$ ). Thus, to keep all the solutions we have to strengthen the slope 
of this line at its smallest extremum. It follows that

$$
L 2_{\mathbb{F}} \equiv \begin{cases}\triangle(\underline{x}+\bar{x}) x-y-\nabla(\underline{x} \bar{x}) \geq 0 & \text { if } \underline{x} \geq 0, \\ \nabla(\underline{x}+\bar{x}) x-y-\nabla(\underline{x} \bar{x}) \geq 0 & \text { if } \bar{x}<0, \\ \triangle(\underline{x}+\bar{x}) x-y & \\ \quad-\nabla(\underline{x} \bar{x}+U l p(\triangle(\underline{x}+\bar{x})) \underline{x}) \geq 0 & \text { if } \bar{x}>0, \underline{x}<0,|\underline{x}| \leq|\bar{x}|, \\ \nabla(\underline{x}+\bar{x}) x-y & \\ \quad-\nabla(\underline{x} \bar{x}-\triangle(U l p(\triangle(\underline{x}+\bar{x})) \bar{x})) \geq 0 & \text { if } \bar{x}>0, \underline{x}<0,|\underline{x}|>|\bar{x}|,\end{cases}
$$

where $U l p(x)$ computes the distance between $x$ and the float following $x$.

(c) Approximation of BIL1,BIL2,BIL3, and BIL4. The general form of BIL1, $B I L 2, B I L 3$, and BIL4 is $x_{i} x_{j}+s_{1} b_{1} x_{i}+s_{2} b_{2} x_{j}+s_{3} b_{1} b_{2} \geq 0$, where $b_{1}$ and $b_{2}$ are floating point numbers corresponding to bounds of $x_{i}$ and $x_{j}$ whereas $s_{i} \in\{-1,1\}$.

The term $s_{3} b_{1} b_{2}$ is the only term which results from a computation: all the other terms use constants which are not subject to round-off errors. Thus, these linear constraints can be rewritten in the following form: $Y+s_{3} b_{1} b_{2}$.

A rounding of $s_{3} b_{1} b_{2}$ towards $+\infty$ enlarges the solution space, and thus ensures that all these linear constraints are safe approximations of $x^{2}$.

It follows that $B I L\{1, \ldots, 4\}_{\mathbb{F}} \equiv Y+\triangle\left(s_{3} b_{1} b_{2}\right) \geq 0$.

(d) Approximation of multivariate linearizations. We are now in the position to introduce the corrections of multivariate linearizations as introduced for the power of $x$. Such linearizations could be rewritten in the following form:

$$
\sum_{i=1}^{n} a_{i} x_{i}+b \geq 0
$$

where $a_{i}$ denotes the expression used to compute the coefficient of variable $x_{i}$, and $b$ is the expression used to compute the constant value. Proposition 7.1 takes advantage of interval arithmetic to compute a safe linearization with coefficients over the floating point numbers.

Proposition 7.1.

$$
\sum_{i=1}^{n} \bar{a}_{i} x_{i}+\sup \left(\bar{b}+\sum_{i=1}^{n} \sup \left(\sup \left(\mathbf{a}_{i} \underline{x}_{i}\right)-\bar{a}_{i} \underline{x}_{i}\right)\right) \geq \sum_{i=1}^{n} a_{i} x_{i}+b \geq 0 \quad \text { for all } x_{i} \in \mathbf{x}_{i} .
$$

Proof. For all $x_{i} \in \mathbf{x}_{i}$, we have

$$
\sum_{i=1}^{n} \bar{a}_{i} x_{i}+\sup \left(\bar{b}+\sum_{i=1}^{n} \sup \left(\sup \left(\mathbf{a}_{i} \underline{x}_{i}\right)-\bar{a}_{i} \underline{x}_{i}\right)\right) \geq \sum_{i=1}^{n} \bar{a}_{i} x_{i}+b+\sum_{i=1}^{n}\left(\sup \left(\mathbf{a}_{i} \underline{x}_{i}\right)-\bar{a}_{i} \underline{x}_{i}\right)
$$

and

$$
\sum_{i=1}^{n} \bar{a}_{i} x_{i}+b+\sum_{i=1}^{n}\left(\sup \left(\mathbf{a}_{i} \underline{x}_{i}\right)-\bar{a}_{i} \underline{x}_{i}\right)=\sum_{i=1}^{n}\left(\bar{a}_{i}\left(x_{i}-\underline{x}_{i}\right)+\sup \left(\mathbf{a}_{i} \underline{x}_{i}\right)\right)+b .
$$

As for all $i \in\{1, \ldots, n\}$, we have $\bar{a}_{i} \geq a_{i}, \sup \left(\mathbf{a}_{i} \underline{x}_{i}\right) \geq a_{i} \underline{x}_{i}$, and for all $x_{i} \in \mathbf{x}_{i}$, $x_{i}-\underline{x}_{i} \geq 0$. Therefore,

$$
\sum_{i=1}^{n}\left(\bar{a}_{i}\left(x_{i}-\underline{x}_{i}\right)+\sup \left(\mathbf{a}_{i} \underline{x}_{i}\right)\right)+b \geq \sum_{i=1}^{n}\left(a_{i}\left(x_{i}-\underline{x}_{i}\right)+a_{i} \underline{x}_{i}\right)+b=\sum_{i=1}^{n} a_{i} x_{i}+b .
$$


This proposition provides a safe approximation of a multivariate linearization which holds for any $a_{i}, x_{i}$, and $b$. This result could be refined by means of the previous approximations. For instance, whenever $\underline{x}_{i} \geq 0, \bar{a}_{i} x_{i} \geq a_{i} x_{i}$. In this case, there is no need for an additional correction.

(e) Approximation of initial constant values. Initial constant values are real numbers that may not have an exact representation within the set of floating point numbers. Thus, a safe approximation is required.

Constant values in inequalities have to be correctly rounded according to the orientation of the inequality. The result presented in the previous paragraph sets the rounding directions which have to be used.

Equations must be transformed into inequalities when their constant values have to be approximated.

7.2. Computation of safe bounds with linear programming algorithm. Linear programming methods can solve problems of the following form:

$$
\begin{aligned}
\min & C^{T} X \\
\text { such that } & \underline{B} \leq A X \leq \bar{B} \\
\text { and } & \underline{X} \leq X \leq \bar{X} .
\end{aligned}
$$

The solution of such a problem is a vector $X_{r} \in \mathbb{R}^{n}$. However, the solution computed by solvers like CPLEX or SOPLEX is a vector $X_{f} \in \mathbb{F}^{n}$ that may be different from $X_{r}$ due to the rounding errors. More precisely, $X_{f}$ is safe for the objective only if $C^{T} X_{r} \geq C^{T} X_{f}$.

Neumaier and Shcherbina [42] provide a cheap method to obtain a rigorous bound of the objective and certificates of infeasibility. The essential observation is that the dual of (7.1) is

$$
\begin{array}{cl}
\max & B^{T} Z^{\prime}+\bar{B}^{T} Z^{\prime \prime} \\
\text { such that } & A^{T}\left(Z^{\prime}-Z^{\prime \prime}\right)=C .
\end{array}
$$

Let $Y=Z^{\prime}-Z^{\prime \prime}$, and let the residue $R=A^{T} Y-C \in \mathbf{R}=[\underline{R}, \bar{R}]$. It follows that

$$
C^{T} X=\left(A^{T} Y-R\right)^{T} X=Y^{T} A X-R^{T} X \in Y^{T}[\underline{B}, \bar{B}]-\mathbf{R}^{T}[\underline{X}, \bar{X}]
$$

and the value of $\mu$, the lower bound of the value of the objective function, is

$$
\mu=\inf \left(Y^{T} \mathbf{B}-\mathbf{R}^{T} \mathbf{X}\right)=\nabla\left(Y^{T} \mathbf{B}-\mathbf{R}^{T} \mathbf{X}\right) .
$$

Formula (7.3) is trivially correct by construction. Note that the precision of such a safe bound depends on the width of the intervals $[\underline{X}, \bar{X}]$.

So, we just have to apply this correction before updating the lower and the upper bounds of each variable.

However, the linear program (7.1) may be infeasible. In that case, Neumaier and Shcherbina show that whenever $d=\inf \left(\mathbf{R}^{\prime T} \mathbf{X}-Y^{T} \mathbf{B}\right)>0$, where $R^{\prime}=A^{T} Y \in \mathbf{R}^{\prime}$, then it is certain that no feasible point exists. However, the precision of interval arithmetic does not always allow us to get a positive value for $d$ while the linear program is actually infeasible. In the latter case, we consider it as feasible. Note that box-consistency may be able to reject most, if not all, of the domains of such variables. 
8. Experimental results. This section reports experimental results of Quad on a variety of twenty standard benchmarks. Benchmarks eco6, katsura5, katsura6, katsura7, tangets2, ipp, assur44, cyclic5, tangents0, chemequ, noon5, geneig, kinema, reimer5, and camera1s were taken from Verschelde's web site, ${ }^{10} \mathrm{kin} 2$ from [60], didrit from [15], lee from [29], and finally yama194, yama195, and yama196 from [63]. The most challenging benchmark is stewgou40 [16]. It describes the 40 possible positions of a Gough-Stewart platform as a function of the values of the actuators. The proposed modelling of this problem consists of 9 equations with 9 variables.

The experimental results are reported in Tables 8.1 and 8.2. Column $n$ (resp., $\delta$ ) shows the number of variables (resp., the maximum polynomial degree). BP $(\Phi)$ stands for a Branch and Prune solver based on the $\Phi$ filtering algorithm, that is to say, a search-tree exploration where a filtering technique $\Phi$ is applied at each node. quad $(\mathrm{H})$ denotes the Quad algorithm where bilinear terms are relaxed with formulae (6.2), power terms with formulae (6.5), and product terms with the quadrification method; $\mathrm{H}$ stands for the heuristic used for decomposing terms in the quadrification process.

The performances of the following five solvers have been investigated.

1. RealPaver: a free Branch and Prune solver ${ }^{11}$ that dynamically combines optimized implementations of box-consistency filtering and 2b-consistency filtering algorithms [5].

2. BP(box): a Branch and Prune solver based on the ILOG $^{12}$ commercial implementation of box-consistency.

3. BP(box+simplex): a Branch and Prune solver based on box and a simple linearization of the whole system without introducing linear relaxations of the nonlinear terms.

4. BP(box+quad (Qmid)): a Branch and Prune solver which combines box and the Quad algorithm where product terms are relaxed with the Qmid heuristic.

5. BP(box+quad(rAI)): a Branch and Prune solver which combines box and the Quad algorithm where product terms are relaxed with the rAI heuristic.

Note that the BP(box+simplex) solver implements a strategy that is slightly different from the approach of Yamamura, Kawata, and Tokue [63].

All the solvers have been parameterized to get solutions or boxes with precision of $10^{-8}$. That is to say, the width of the computed intervals is smaller than $10^{-8}$. A solution is said to be safe if we can prove its uniqueness within the considered box. This proof is based on the well-known Brouwer fixed point theorem (see [20]) and requires just a single test.

Sols, Ksplit, and $T(s)$ are, respectively, the number of solutions, the number of thousands of branchings (or splittings), and the execution time in seconds. The number of solutions is followed with a number of safe solutions between brackets. A "-" in the column $T(s)$ means that the solver was unable to find all the solutions within eight hours. All the computations have been performed on a PC with Pentium IV processor at $2.66 \mathrm{GHz}$ running Linux. The compiler was GCC 2.9.6 used with the -O6 optimization flag.

The performances of RealPaver, BP(box), and BP(box+quad(Qmid)) are displayed in Table 8.1. The benchmarks have been grouped into three sets. The first

\footnotetext{
${ }^{10}$ The database of polynomial systems is available at http://www.math.uic.edu/ jan/Demo/.

${ }^{11}$ See http://www.sciences.univ-nantes.fr/info/perso/permanents/granvil/realpaver/main.html.

${ }^{12}$ See http://www.ilog.com/products/jsolver.
} 
TABLE 8.1

Experimental results: comparing Quad and Constraint solvers.

\begin{tabular}{|l||r|l||r|r|r||r|r|r||r|r|}
\hline \multicolumn{2}{l|}{} & & & \multicolumn{2}{c|}{ BP(box+quad(Qmid)) } & \multicolumn{3}{|c||}{ BP(box) } & \multicolumn{2}{|c|}{ Realpaver } \\
\hline Name & $n$ & $\delta$ & Sols & Ksplits & $T(s)$ & Sols & Ksplits & $T(s)$ & Sols & $T(s)$ \\
\hline \hline cyclic5 & 5 & 5 & $10(10)$ & 0.6 & 45.8 & $10(10)$ & 13.4 & 26.3 & 10 & 291.6 \\
eco6 & 6 & 3 & $4(4)$ & 0.4 & 15.3 & $4(4)$ & 1.7 & 3.7 & 4 & 1.3 \\
\hline \hline assur44 & 8 & 3 & $10(10)$ & 0.1 & 49.5 & $10(10)$ & 15.8 & 72.5 & 10 & 72.6 \\
ipp & 8 & 2 & $10(10)$ & 0.0 & 5.7 & $10(10)$ & 4.6 & 14.0 & 10 & 16.8 \\
katsura5 & 6 & 2 & $16(11)$ & 0.1 & 9.9 & $41(11)$ & 8.2 & 12.7 & 12 & 6.7 \\
katsura6 & 7 & 2 & $60(24)$ & 0.5 & 121.9 & $182(24)$ & 136.6 & 281.4 & 32 & 191.8 \\
kin2 & 8 & 2 & $10(10)$ & 0.0 & 6.2 & $10(10)$ & 3.5 & 19.3 & 10 & 2.6 \\
noon5 & 5 & 3 & $11(11)$ & 0.1 & 17.9 & $11(11)$ & 50.2 & 58.7 & 11 & 39.0 \\
tangents2 & 6 & 2 & $24(24)$ & 0.1 & 17.5 & $24(24)$ & 14.1 & 27.9 & 24 & 16.5 \\
\hline \hline camera1s & 6 & 2 & $16(16)$ & 1.0 & 28.9 & $2(2)$ & 11820.3 & - & 0 & - \\
didrit & 9 & 2 & $4(4)$ & 0.1 & 14.7 & $4(4)$ & 51.3 & 132.9 & 4 & 94.6 \\
geneig & 6 & 3 & $10(10)$ & 0.8 & 39.1 & $10(10)$ & 290.7 & 868.6 & 10 & 475.6 \\
kinema & 9 & 2 & $8(8)$ & 0.2 & 19.9 & $15(7)$ & 244.0 & 572.4 & 8 & 268.4 \\
katsura7 & 8 & 2 & $58(42)$ & 1.7 & 686.9 & $231(42)$ & 1858.5 & 11104.1 & 44 & 4671.1 \\
lee & 9 & 2 & $4(4)$ & 0.5 & 43.3 & $0(0)$ & 8286.3 & - & 0 & - \\
reimer5 & 5 & 6 & $24(24)$ & 0.1 & 53.0 & $24(24)$ & 2230.2 & 2892.5 & 24 & 733.9 \\
stewgou40 & 9 & 4 & $40(40)$ & 1.6 & 924.0 & $11(11)$ & 3128.6 & - & 8 & - \\
yama194 & 16 & 3 & $9(9)$ & 0.0 & 11.1 & $9(8)$ & 1842.1 & - & 0 & - \\
yama195 & 60 & 3 & $3(3)$ & 0.0 & 106.1 & $0(0)$ & 19.6 & - & 0 & - \\
yama196 & 30 & 1 & $2(1)$ & 0.0 & 6.7 & $0(0)$ & 816.7 & - & 0 & - \\
\hline
\end{tabular}

group contains problems where the QuadSolver does not behave very well. These problems are quite easy to solve and the overhead of the relaxation and the calls to a linear solver does not pay off. The second group contains a set of benchmarks for which the QuadSolver compares well with the two other constraint solvers: the QuadSolver requires always much less splitting and often less time than the other solvers. In the third group, which contains difficult problems, the QuadSolver outperforms the two other constraint solvers. The latter were unable to solve most of these problems within eight hours whereas the QuadSolver managed to find all the solutions for all but two of them in less than 8 minutes. For instance, BP(box) requires about 74 hours to find the four solutions of the Lee benchmark whereas the QuadSolver managed to do the job in a couple of minutes. Likewise, the QuadSolver did find the forty safe solutions of the stewgou 40 benchmark in about 15 minutes whereas BP(box) required about 400 hours. The essential observation is that the QuadSolver spends more time in the filtering step but it performs much less splitting than classical solvers. This strategy pays off for difficult problems.

All the problems, except cyclic5 and reimer5, contain many quadratic terms and some product and power terms. cyclic5 is a pure multilinear problem that contains only sums of products of variables. The Quad algorithm has not been very efficient for handling this problem. Of course, one could not expect an outstanding performance on this bench since product term relaxation is a poor approximation of multilinear terms. reimer5 is a pure power problem of degree 6 that has been well solved by the Quad algorithm.

Table 8.2 displays the performances of solvers combining box-consistency and three different relaxation techniques. There is no significant difference between the solver based on the Qmid heuristics and the solver based on the rAI heuristics. Indeed, both heuristics provide convex and concave envelopes of the product terms. The QuadSolver with relaxations outperforms the BP (box+simplex) approach for all 
TABLE 8.2

Experimental results: comparing Quad based on different relaxations.

\begin{tabular}{|l||r|r|r||r|r|r||r|r|r|}
\hline \multicolumn{1}{|c||}{} & \multicolumn{3}{c|}{ BP(box+simplex) } & \multicolumn{2}{c||}{ BP(box+quad(Qmid)) } & \multicolumn{2}{c|}{ BP(box+quad(rAI)) } \\
\hline Name & Sols & Ksplits & $T(s)$ & Sols & Ksplits & $T(s)$ & Sols & Ksplits & $T(s)$ \\
\hline \hline cyclic5 & $10(10)$ & 15.6 & 60.6 & $10(10)$ & 0.6 & 45.8 & $10(10)$ & 0.8 & 76.1 \\
eco6 & $4(4)$ & 1.1 & 7.2 & $4(4)$ & 0.4 & 15.3 & $4(4)$ & 0.4 & 15.3 \\
\hline \hline assur44 & $10(10)$ & 15.5 & 261.9 & $10(10)$ & 0.1 & 49.5 & $10(10)$ & 0.1 & 50.0 \\
ipp & $10(10)$ & 3.2 & 39.7 & $10(10)$ & 0.0 & 5.7 & $10(10)$ & 0.0 & 5.7 \\
katsura5 & $41(11)$ & 7.7 & 47.8 & $16(11)$ & 0.1 & 9.9 & $16(11)$ & 0.1 & 9.9 \\
katsura6 & $182(24)$ & 135.2 & 1156.7 & $60(24)$ & 0.5 & 121.9 & $60(24)$ & 0.5 & 122.7 \\
kin2 & $10(10)$ & 3.4 & 42.5 & $10(10)$ & 0.0 & 6.2 & $10(10)$ & 0.0 & 6.2 \\
noon5 & $11(11)$ & 49.6 & 226.7 & $11(11)$ & 0.1 & 17.9 & $11(11)$ & 0.1 & 17.8 \\
tangents2 & $24(24)$ & 11.4 & 77.7 & $24(24)$ & 0.1 & 17.5 & $24(24)$ & 0.1 & 17.5 \\
\hline \hline camera1s & $4(4)$ & 3298.6 & - & $16(16)$ & 1.0 & 28.9 & $16(16)$ & 1.0 & 29.9 \\
didrit & $4(4)$ & 5.3 & 93.2 & $4(4)$ & 0.1 & 14.7 & $4(4)$ & 0.1 & 14.7 \\
geneig & $10(10)$ & 202.8 & 2036.8 & $10(10)$ & 0.8 & 39.1 & $10(10)$ & 0.8 & 39.2 \\
kinema & $13(7)$ & 87.0 & 1135.1 & $8(8)$ & 0.2 & 19.9 & $8(8)$ & 0.2 & 20.0 \\
katsura7 & $231(42)$ & 1867.2 & 21679.6 & $58(42)$ & 1.7 & 686.9 & $58(42)$ & 1.7 & 684.0 \\
lee & $2(2)$ & 78.1 & 1791.8 & $2(2)$ & 0.3 & 27.1 & $2(2)$ & 0.3 & 26.5 \\
lee2 & $4(4)$ & 117.6 & 2687.2 & $4(4)$ & 0.5 & 43.3 & $4(4)$ & 0.5 & 43.3 \\
reimer5 & $24(24)$ & 2208.7 & 10433.5 & $24(24)$ & 0.1 & 53.0 & $24(24)$ & 0.1 & 53.1 \\
stewgou40 & $13(13)$ & 716.3 & - & $40(40)$ & 1.6 & 924.0 & $40(40)$ & 1.5 & 914.1 \\
yama194 & $9(7)$ & 442.0 & - & $9(9)$ & 0.0 & 11.1 & $9(9)$ & 0.0 & 11.2 \\
yama195 & $3(2)$ & 0.0 & 37.7 & $3(3)$ & 0.0 & 106.1 & $3(3)$ & 0.0 & 106.7 \\
yama196 & $2(1)$ & 0.0 & 6.6 & $2(1)$ & 0.0 & 6.7 & $2(1)$ & 0.0 & 6.7 \\
\hline
\end{tabular}

benchmarks but yama195, which is a quasilinear problem. These performances on difficult problems illustrate well the capabilities of the relaxations.

Note that Verschelde's homotopy continuation system, PHCpack [62], required $115 \mathrm{~s}$ to solve lee and $1047 \mathrm{~s}$ to solve stewgou40 on our computer. PHCpack is a state-of-the-art system in solving polynomial systems of equations. Unfortunately, it is limited to polynomial systems and does not handle inequalities. PHCpack searches for all the roots of the equations, whether real or complex, and it does not restrict its search to a given subspace. The homotopy continuation approach also suffers from an exponential growing computation time which depends on the number of nonlinear terms (PHCpack failed to solve yama195 which contains 3600 nonlinear terms). In contrast to homotopy continuation methods, QuadSolver can easily be extended to nonpolynomial systems.

Thanks to Arnold Neumaier and Oleg Shcherbina, we had the opportunity to test BARON [50] with some of our benchmarks. QuadSolver compares well with this system. For example, BARON $6.0^{13}$ and QuadSolver require more or less the same time to solve camera1s, didrit, kinema, and lee. BARON needs only $1.59 \mathrm{~s}$ to find all the solutions of yama196 but it requires $859.6 \mathrm{~s}$ to solve yama195. Moreover, BARON loses some solutions on reimer5 (22 solutions found) and stewgou40 (14 solutions found) whereas it generates numerous wrong solutions for these two problems. We must also underline that BARON is a global optimization problem solver and that it has not been built to find all the solutions of a problem.

9. Conclusion. In this paper, we have exploited an RLT schema to take into account specific semantics of nonlinear terms. This relaxation process is incorporated in the Branch and Prune process [60] that exploits interval analysis and constraint satis-

\footnotetext{
${ }^{13}$ The tests were performed on an Athlon XP 1800 computer.
} 
faction techniques to find rigorously all solutions in a given box. The reported experimental results show that this approach outperforms the classical constraint solvers.

Pesant and Boyer [44, 45] first introduced linear relaxations in a CLP language to handle geometrical constraints. However, the approximation of the constraints was rather weak. The approach introduced in this paper is also related to recent work that has been done in the interval analysis community as well as to some work achieved in the optimization community.

In the interval analysis community, Yamamura, Kawata, and Tokue [63] used a simple linear relaxation procedure where nonlinear terms are replaced by new variables to prove that some box does not contain solutions. No convex/concave outerestimations are proposed to obtain a better approximation of the nonlinear terms. As pointed out by Yamamura, Kawata, and Tokue, this approach is well adapted to quasi-linear problems: "This test is much more powerful than the conventional test if the system of nonlinear equations consists of many linear terms and a relatively small number of nonlinear terms" [63].

The global optimization community also worked on solving nonlinear equation problems by transforming them into an optimization problem (see, for example, Chapter 23 in [17]). The optimization approach has the capability to take into account specific semantics of nonlinear terms by generating a tight outer-estimation of these terms. The pure optimization methods are usually not rigorous since they do not take into account rounding errors and do not prove the uniqueness of the solutions found.

Acknowledgments. We thank Arnold Neumaier for his fruitful comments on an early version of this paper. We are also grateful to Arnold Neumaier and Oleg Shcherbina for their help in testing BARON.

\section{REFERENCES}

[1] F. A. Al-Khayyal and J. E. Falk, Jointly constrained biconvex programming, Math. Oper. Res., 8 (1983), pp. 273-286.

[2] E. Allgower and K. Georg, Numerical Continuation Methods: An Introduction, SpringerVerlag, Berlin, 1990.

[3] C. Audet, P. Hansen, B. Jaumard, And G. Savard, Branch and cut algorithm for nonconvex quadratically constrained quadratic programming, Math. Program., 87 (2000), pp. 131-152.

[4] A. B. Babichev, O. P. Kadyrova, T. P. Kashevarova, A. S. Leshchenko, and A. L. Semenov, Unicalc, a novel approach to solving systems of algebraic equations, Interval Computations, 2 (1993), pp. 29-47.

[5] F. Benhamou, F. Gounlard, L. Granvilliers, and J.-F. Puget, Revising hull and box consistency, in Proceedings of ICLP '99, 1999, MIT Press, pp. 230-244.

[6] F. Benhamou, D. McAllester, and P. Van-Hentenryck, CLP(intervals) revisited, in Proceedings of the International Symposium on Logic Programming, MIT Press, Cambridge, MA, 1994, pp. 124-138.

[7] F. Benhamou and W. Older, Applying interval arithmetic to real, integer and Boolean constraints, J. Logic Programming, 32 (1997), pp. 1-24.

[8] B. Botella And P. Taillibert, Interlog: Constraint logic programming on numeric intervals, in 3rd International Workshop on Software Engineering, Artificial Intelligence and Expert Systems, Oberammergau, 1993.

[9] R. L. Burden and J. D. Faires, Numerical Analysis, 5th ed., PWS-KENT, Boston, MA, 1993.

[10] J. C. Cleary, Logical arithmetic, Future Computing Systems, 2 (1987), pp. 125-149.

[11] H. Collavizza, F. Delobel, And M. Rueher, Comparing partial consistencies, Reliable Computing, 5 (1999), pp. 213-228.

[12] A. Colmerauer, Spécifications de Prolog IV, Technical report, GIA, Faculté des Sciences de Luminy, France, 1994.

[13] D. Cox, J. Little, And D. O'Shea, Ideals, Varieties, and Algorithms, 2nd ed., SpringerVerlag, New York, 1997. 
[14] Y. Crama, Recognition problems for polynomial in 0-1 variables, Math. Progr., 44 (1989), pp. $139-155$.

[15] O. DidRIT, Analyse par Intervalles pour L'automatique: Résolution Globale et Garantie de Problèmes Non Linéaires en Robotique et en Commande Robuste, Ph.D. thesis, Université Parix XI Orsay, France, 1997.

[16] P. Dietmaier, The Stewart-Gough platform of general geometry can have 40 real postures, in Advances in Robot Kinematics: Analysis and Control, Kluwer, Dordrecht, 1998, pp. 1-10.

[17] C. A. Floudas, ED., Deterministic Global Optimization: Theory, Algorithms and Applications, Kluwer, Dordrecht, 2000.

[18] D. GoldBerg, What every computer scientist should know about floating-point arithmetic, ACM Computing Surveys, 23 (1991), pp. 5-48.

[19] E. Hansen AND S. Sengupta, Bounding solutions of systems of equations using interval analysis, BIT, 21 (1981), pp. 203-221.

[20] E. R. HAnsen, Global Optimization Using Interval Analysis, Marcel Dekker, New York, 1992.

[21] H. Hong And V. Stahl, Starting regions by fixed points and tightening, Computing, 53 (1994), pp. 323-335.

[22] ILOG Solver 4.0, Reference Manual, ILOG, Mountain View, 1997.

[23] R. B. Kearfott, Tests of generalized bisection, ACM Trans. Math. Software, 13 (1987), pp. $197-220$.

[24] R. KRAWCZYK, Newton-Algorithmen zur Bestimmung von Nullstellen mit Fehlerschranken, Computing, 4 (1969), pp. 187-201.

[25] V. Kreinovich, A. Lakeyev, J. Rohn, and P. Kahl, Computational Complexity and Feasibility of Data Processing and Interval Computations, Kluwer, Dordrecht, 1998.

[26] Y. Lebbah and O. Lhomme, Accelerating filtering techniques for numeric CSPs, Artificial Intelligence, 139 (2002), pp. 109-132.

[27] Y. Lebbah, M. Rueher, And C. Michel, A global filtering algorithm for handling systems of quadratic equations and inequations, in Proc. of the 8th International Conference on Principles and Practice of Constraint Programming, Cornell University, New York, 2002, Lecture Notes in Comput. Sci. 2470, pp. 109-123.

[28] J. H. M. Lee and M. H. van Emden, Interval computation as deduction in CHIP, J. Logic Programming, 16 (1993), pp. 255-276.

[29] T.-Y. Lee And J.-K. Shim, Elimination-based solution method for the forward kinematics of the general Stewart-Gough platform, in Computational Kinematics, F. C. Park and C. C. Iurascu, eds., 2001, pp. 259-267.

[30] O. Lhomme, Consistency techniques for numeric CSPs, in Proceedings of International Joint Conference on Artificial Intelligence, Chambéry, France, 1993, pp. 232-238.

[31] A. Mackworth, Consistency in networks of relations, J. Artificial Intelligence, 8 (1977), pp. $99-118$.

[32] G. P. MCCoRmick, Computability of global solutions to factorable nonconvex programsPart I-Convex underestimating problems, Math. Progr., 10 (1976), pp. 147-175.

[33] C. Michel, Y. Lebbah, And M. Rueher, Safe embedding of the simplex algorithm in a CSP framework, in Proc. of 5th Int. Workshop on Integration of AI and OR Techniques in Constraint Programming for Combinatorial Optimization Problems CPAIOR 2003, CRT, Université de Montréal, 2003, pp. 210-220.

[34] U. Montanari, Networks of constraints: Fundamental properties and applications to image processing, Inform. Sci., 7 (1974), pp. 95-132.

[35] R. Moore, Interval Analysis, Prentice-Hall, Englewood Cliff, NJ, 1966.

[36] R. E. Moore, Methods and Applications of Interval Analysis, SIAM Stud. Appl. Math. 2, SIAM, Philadelphia, 1979.

[37] A. P. MoRgan, Computing all solutions to polynomial systems using homotopy continuation, Appl. Math. Comput., 24 (1987), pp. 115-138.

[38] A. Neumaier, Interval Methods for Systems of Equations, Encyclopedia Math. Appl. 37, Cambridge University Press, Cambridge, 1990.

[39] A. Neumaier, The wrapping effect, ellipsoid arithmetic, stability and confidence region, Comput. Suppl., 9 (1993), pp. 175-190.

[40] A. Neumaier, Introduction to Numerical Analysis, Cambridge University Press, Cambridge, 2001.

[41] A. Neumaier, Complete search in continuous global optimization and constraint satisfaction, in Acta Numerica 2004, A. Iserles, ed., Cambridge University Press, Cambridge, UK, 2004, pp. 271-369.

[42] A. Neumaier And O. Shcherbina, Safe bounds in linear and mixed-integer programming, Math. Progr. A, 99 (2004), pp. 283-296. 
[43] W. J. Older And A. Velino, Extending prolog with constraint arithmetic on real intervals, in Proc. of IEEE Canadian Conference on Electrical and Computer Engineering, IEEE Computer Society Press, 1990, pp. 14.1.1-14.1.4.

[44] G. Pesant And M. Boyer, QUAD-CLP(R): Adding the power of quadratic constraints, in Principles and Practice of Constraint Programming '94, Lecture Notes in Comput. Sci. 874, 1994, pp. 95-107.

[45] G. Pesant and M. Boyer, Reasoning about solids using constraint logic programming, J. Automat. Reason., 22 (1999), pp. 241-262.

[46] H. Ratschek and J. Rokne, Computer Methods for the Range of Functions, Ellis Horwood Ser. Math. Appl., Ellis Horwood, New York, 1984.

[47] N. Revol And F. Roulllier, Motivations for an arbitrary precision interval arithmetic and the MPFI library, in Workshop on Validated Computing, Toronto, Canada, 2002.

[48] A. Rikun, A convex envelope formula for multilinear functions, J. Global Optim., 10 (1997), pp. $425-437$.

[49] H. S. Ryoo AND N. V. SAhinidis, Analysis of bounds for multilinear functions, J. Global Optim., 19 (2001), pp. 403-424.

[50] N. V. Sahinidis AND M. Twarmalani, BARON 5.0: Global Optimization of Mixed-Integer Nonlinear Programs, Technical report, Department of Chemical and Biomolecular Engineering, University of Illinois at Urbana-Champaign, 2002.

[51] N. V. Sahinidis And M. Twarmalani, Global optimization of mixed-integer programs: A theoretical and computational study, Math. Progr., 99 (2004), pp. 563-591.

[52] H. D. Sherali, Convex envelopes of multilinear functions over a unit hypercube and over special discrete sets, Acta Math. Vietnam., 22 (1997), pp. 245-270.

[53] H. D. Sherali and W. P. Adams, A Reformulation-Linearization Technique for Solving Discrete and Continuous Nonconvex Problems, Kluwer, Dordrecht, 1999.

[54] H. D. Sherali And C. H. Tuncbilek, A global optimization algorithm for polynomial using a reformulation-linearization technique, J. Global Optim., 2 (1992), pp. 101-112.

[55] H. D. Sherali and C. H. Tuncbilek, A reformulation-convexification approach for solving nonconvex quadratic programming problems, J. Global Optim., 7 (1995), pp. 1-31.

[56] H. D. Sherali and C. H. Tuncbilek, A comparison of two reformulation-linearization technique based on linear programming relaxations for polynomial porgramming problems, J. Global Optim., 10 (1997), pp. 381-390.

[57] H. D. Sherali And C. H. TuncBilek, New reformulation linearization/convexification relaxations for univariate and multivariate polynomial programming problems, Oper. Res. Lett., 21 (1997), pp. 1-9.

[58] N. Z. Shor, Dual quadratic estimates in polynomial and Boolean programming, Ann. Oper. Res., 25 (1990), pp. 163-168.

[59] M. Tawarmalani and N. V. Sahinidis, eds., Convexification and Global Optimization in Continuous and Mixed-Integer Non-Linear Programming, Kluwer, Dordrecht, 2002.

[60] P. Van Hentenryck, D. McAllester, And D. Kapur, Solving polynomial systems using a branch and prune approach, SIAM J. Numer. Anal., 34 (1997), pp. 797-827.

[61] P. Van-Hentenryck, L. Michel, and Y. Deville, Numerica: A Modeling Language for Global Optimization, MIT Press, Cambridge, MA, 1997.

[62] J. Verschelde, Algorithm 795: PHCPACK: A general-purpose solver for polynomial systems by homotopy continuation, ACM Trans. Math. Software, 25 (1999), pp. 251-276.

[63] K. Yamamura, H. Kawata, AND A. TokUe, Interval solution of nonlinear equations using linear programming, BIT, 38 (1998), pp. 186-199. 